\title{
Infinity-Norm Rotation Transforms
}

\author{
Lei Yang and Pengwei Hao, Member, IEEE
}

\begin{abstract}
A new general paradigm of dynamic-range-preserving one-to-one mapping-infinity-norm rotations, analogous to the general 2-norm rotations, are proposed in this paper. Analogous to the well-known discrete cosine transforms, the linear 2-norm rotation transforms which preserve the 2-norm of the rotated vectors, the proposed infinity-norm rotation transforms are piecewise linear transforms which preserve the infinity-norm of vectors. Besides the advantages of perfect reversibility, in-place calculation and dynamic range preservation, the infinity-norm rotation transforms also have good energy-compact ability, which is suitable for signal compression and analysis. It can be implemented by shear transforms based on the 2-D rotation factorization of similar orthogonal transform matrices, such as DCT matrices. The performance of the new transforms is illustrated with 2-D patterns and histograms. Its good performance in lossy and lossless image compression, compared with other integer reversible transforms, is demonstrated in the experiments.
\end{abstract}

Index Terms-Discrete cosine transform, dynamic range, infinity-norm rotation, integer reversible transform, transform coding.

\section{INTRODUCTION}

$\mathbf{L}$ INEAR transforms, such as Fourier transforms, discrete cosine transforms and wavelet transforms, have wide applications in signal processing, with the ability of energycompact frequency decomposition. For prevalent digital images and videos, input signals are available as integer data sequences, or more generally as fixed-point data sequences. In some special applications such as military, medical and remote sensing imaging [15], loss of information is not tolerated during processing. Therefore, integer reversible transforms are highly desirable. However, these linear transforms cannot achieve perfect reversibility directly due to the precision limitation of computers. In the literature of transforms, the indirectly implemented transforms usually refer to integer discrete wavelet transforms (IntDWT), integer discrete cosine transforms (IntDCT), integer discrete Fourier transforms (IntDFT) and so forth.

Generally speaking, the integer reversible transforms are the marriage of corresponding linear transforms and an appropriate

Manuscript received March 05, 2008; accepted January 12, 2009. First published March 21, 2009; current version published June 17, 2009. The associate editor coordinating the review of this manuscript and approving it for publication was Prof. William A. Sethares. This work was supported by NSFC of China under Grant 60572043 and NKBRPC of China under Grant 2004CB318005.

L. Yang was with the Center for Information Science, Peking University, Beijing 100871, China. She is now with the Multimedia Communications and Networking Laboratory, the Department of Electrical and Computer Engineering, University of Florida, Gainesville, FL 32611 USA (e-mail: leiyang@uff.edu).

P. Hao is with the Department of Computer Science, Queen Mary, University of London, London E1 4NS, U.K., and with the Center for Information Science, Peking University, Beijing 100871, China (e-mail: phao@dcs.quml.ac.uk; phao@cis.pku.edu.cn).

Color versions of one or more of the figures in this paper are available online at http://ieeexplore.ieee.org.

Digital Object Identifier 10.1109/TSP.2009.2018631 reversible transform framework with rounding operations in an attempt to approximate the corresponding linear transforms. Sweldens et al. [1], [5], [6] first proposed factoring wavelets into lifting steps to realize integer transformation. With the lifting scheme, a new generation of wavelets was constructed, calculated in-place and with further reduced computational complexity. Chen [7] and Liang et al. [8] combined the lifting framework with discrete cosine transforms to construct fast integer DCT, and Oraintara et al. [9] proposed fast integer Fourier transforms (IntFFT) which are also based on the lifting scheme. Besides, another integer transform framework-overlapping rounding transform (ORT) — was developed by Jung and Prost [4], which is, later proved by Adams [10], equivalent to a special case of lifting with only trivial extensions. For generic linear transforms, the elementary reversible matrices with Gaussian integer units as the diagonal entries were proposed by Hao and Shi [3] for triangular matrix factorization to realize integer-to-integer transforms. A linear transform with unitary determinant was further proved to be integer reversible if a PLUS factorization was applied to the transform matrix [2]. Differently, Plonka [21] chose expansion factors for transforms to expand the ranges of the transforms larger than their input domains, and this transform domain redundancy was utilized for reversibility. Modulo transforms (MT), an alternative to lifting, was proposed by Srinivasan [19] recently, which employed certain Pythagorean triples that could be critically quantized to produce a reversible, normalized, scale-free transform, and the ladder structure was employed to approximate orthonormal transforms by 2-point rotations.

For the linear transforms, the bit width of the low-frequent coefficients is generally greater than that of the original data, so the dynamic ranges of the transforms are expanded [11]. For example, the discrete cosine transform matrix of type II of order $n$ is

$$
\mathrm{C}_{n}^{\mathrm{II}}:=\sqrt{\frac{2}{n}} \varepsilon_{n}(j) \cos \frac{j(2 k+1) \pi}{2 n}
$$

where $\varepsilon_{n}(0):=\sqrt{2} / 2$ and $\varepsilon_{n}(j):=1$ for $j, k \in$ $\{1, \cdots, n-1\}$. It is easy to know $\left\|\mathrm{C}_{n}^{\mathrm{II}}\right\|_{\infty}=\sqrt{n}$. It indicates that $m$-bit inputs result in $m+\log _{2} \sqrt{n}$ bit outputs, i.e., 16-bit memory space is generally needed in programs to store a 9-bit output for each 8-bit input after 4-point DCT transformation with general personal computers. Even more, the corresponding integer reversible transforms, IntDCT, usually need greater expanded ranges to keep reversibility. However, if we encounter a computational environment with only limited buffer, fixed-point arithmetic units and fixed-width channels, or with fixed representing word length, the traditional integer transforms will fail, and the dynamic-range-preserving transforms are desired. They are appealing for saving memory and 
computational resources in both compression and decompression, and attractive for speeding up the computational process of coding, since only are the processing units for fixed-bitwidth operands needed.

The solution to integer reversible and dynamic-range-preserving transformation is a challenging research topic, since the constant dynamic range, the compact coefficient representation and reversibility are conflicting goals. Not much has been done to find the best of all possible solutions, except three methods. Chao et al. [12] utilized the complementary code and modular arithmetic automatically to preserve the dynamic ranges of integer wavelet transforms. However, the application of this approach is limited to lossless compression, in which the large positive transform coefficients become negative and vice versa due to modular arithmetic. Thus, the recovered images suffer severe blocking artifacts and salt-and-pepper noise when transform coefficients are lossily compressed. The other two approaches to avoid the dynamic range expansion is Table-Lookup Haar-like transform (TLHaar) and Piecewise Linear Haar-like transform (PLHaar) proposed by Senecal et al. [13], [14]. TLHaar and PLHaar are two-point transforms, both evolving from the S Transform, the integer realization of Haar wavelet transform. TLHaar needs a dynamic look-up table built by optimized permutation and PLHaar is a special 2-D case of our proposed infinity-norm rotation. In this paper, we construct general integer-reversible and dynamic-range-preserving infinity-norm rotation transforms by analogy with rotations in the 2-norm space (orthogonal transforms, e.g., DCT). Although PLHaar is a special case of our proposed infinity-norm rotation transforms, it is not straightforward to obtain our proposed transforms from PLHaar.

The paper is organized as follows. In Section II, we begin with the infinity-norm rotation, and discuss its properties. In Section III, we further propose the infinity-norm rotation transforms which preserve the constant dynamic range, i.e., keep coefficients the same bitwidth as the original and the reconstructed signals, under the illumination of discrete cosine transforms. Examples and practical issues of implementation are considered. Then, its performance is compared with other transforms in Section IV. Its applications in lossy and lossless image coding are investigated in Section V. The last section comes with the conclusions and the future work.

\section{INFINITY-NORM ROTATION}

A linear orthogonal transform is a rotation in Euclidian space, which preserves the 2-norm of the rotated vectors. Correspondingly, a rotation that preserves the dynamic-range is a rotation in the infinity-norm space, called infinity-norm rotation. We begin with more general notation of $p$-norm rotation that preserves the $p$-norm of the vectors after the rotation.

\section{A. p-Norm Rotation}

Definition 1. $p$-Norm Isometry Set $S_{d}^{p}: S_{d}^{p}$ is the set of vectors $\mathbf{x}=\left(x_{1}, x_{2}, \cdots, x_{n}\right)$, in which the $p$-norms of all the vectors are equal to $d$

$$
S_{d}^{p}=\left\{\mathbf{x} \mid \mathbf{x} \in l^{p} \wedge\|\mathbf{x}\|_{p}=d\right\}
$$

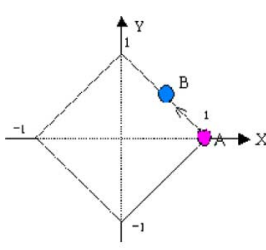

(a)

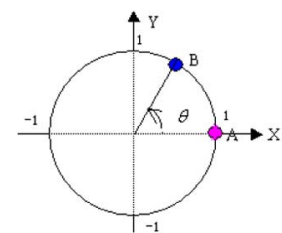

(b)

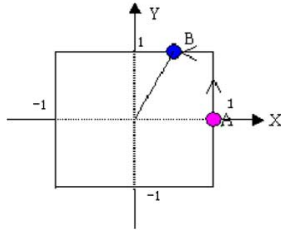

(c)
Fig. 1. Unit $p$-norm rotations in 2-D space: (a) 1-norm rotation, (b) 2-norm rotation, and (c) infinity-norm rotation.

Generally, $S_{d}^{p}$ is called a $p$-norm circle, and $S_{1}^{p}$ is called the unit circle of the $p$-norm isometry set.

Specifically, a 1-norm circle in 2-D space is a rhomboid; a 2-norm (Euclidean norm) circle is a normal circle in 2-D and a sphere in 3-D; equivalently, the infinity-norm circles are squares in 2-D and cubes in 3-D. Generally, in $n$-D space, an infinitynorm circle is a regular convex hyper-polyhedron, of which all the sides are linear in planes or hyperplanes.

Definition 2. p-Norm Rotation: $p$-norm rotation $R_{p}$ is the transform that preserves the $p$-norm of the vectors, and geometrically moves the end points of the vectors in the $p$-norm isometry set $S_{d}^{p}$, i.e.,

$$
R_{p}: S_{d}^{p} \rightarrow S_{d}^{p}, \forall \mathbf{x} \in S_{d}^{p},\|\mathbf{x}\|_{p}=\left\|R_{p}(\mathbf{x})\right\|_{p}=d .
$$

Accordingly, a transform that preserves 1-norm, 2-norm, or the infinity-norm is a 1-norm rotation, 2-norm rotation or an infinity-norm rotation, respectively. These types of rotations in 2-D space are illustrated in Fig. 1. They are performed along the unit circles of 1-norm $\left(S_{1}^{1}\right), 2$-norm $\left(S_{1}^{2}\right)$ and infinity-norm $\left(S_{1}^{\infty}\right)$ counterclockwise with respect to the $X$-axis.

Definition 3. Angle of $p$-Norm rotation: In 2-D space, angle is the amount of $p$-norm rotation between two vectors in $S_{d}^{p}$, which can be measured by the ratio of the runlength sweeping from the first vector to the second vector to the constant $p$-norm radius $d$ :

$$
\theta_{p}=\frac{\text { runlength }}{\text { radius }} .
$$

Particularly, $\theta_{2}=($ arc length $) /$ radius is the angle measure of a 2 -norm rotation, and $\theta_{\infty}=($ runlength $) /($ side length $\left.) / 2\right)$ is that of an infinity-norm rotation. After rotating a complete circle, $\theta_{2}=2 \pi$ and $\theta_{\infty}=8$. The equivalent relation between $\theta_{\infty}$ and $\theta_{2}$ for $\theta_{2} \in[0, \pi / 4]$ can be defined as

$$
\theta_{\infty}=\tan \theta_{2}
$$

Especially, $\theta_{\infty}=1$ when $\theta_{2}=\pi / 4$ and $\theta_{\infty}=2$ when $\theta_{2}=\pi / 2$. Other relations can be found by combining the above rotations. With these angle relations, the infinity-norm rotations are the dynamic-range-preserving approximation of the corresponding linear orthogonal transforms, and can be used as multiple component transforms [18].

\section{B. Properties of Infinity-Norm Rotation}

We use $B=R_{\infty}\left(A, \theta_{\infty}\right)$ to denote the rotation from point $A$ to point $B$ along an infinity-norm isometry set through an angle $\theta_{\infty}$. For the infinity-norm rotation, we have the following 
properties, some of which may also be the properties of other $p$-norm rotations.

1) Composition: Composition of two infinity-norm rotations results in another infinity-norm rotation.

2) Unique inverse: An infinity-norm rotation has a unique inverse rotation, if the runlength is always the shortest.

3) Group of infinity-norm rotations: The set of all infinitynorm rotations of the same center and radius forms a group [22], [25].

4) Constant infinity-norm:

$$
\|\mathbf{x}\|_{\infty}=\left\|R_{\infty}\left(\mathbf{x}, \theta_{\infty}\right)\right\|_{\infty} .
$$

5) Periodicity:

$$
R_{\infty}\left(\mathbf{x}, \theta_{\infty}\right)=R_{\infty}\left(\mathbf{x}, \theta_{\infty}+T\right)
$$

where $T$ is the period of the rotation. In 2-D space, $T=8$.

6) Bijection:

$$
\begin{aligned}
& \text { For } \alpha_{\infty}, \beta_{\infty} \in[0, T), \\
& \qquad R_{\infty}\left(\mathbf{x}, \alpha_{\infty}\right)=R_{\infty}\left(\mathbf{x}, \beta_{\infty}\right) \Leftrightarrow \alpha_{\infty}=\beta_{\infty} .
\end{aligned}
$$

7) Piecewise linearity: As mentioned above, all sides of a infinity-norm isometry set $S_{d}^{p}$ are linear in planes or hyperplanes, so the infinity-norm rotation from one point to another on the same plane of $S_{d}^{p}$ can be expressed as a piece of linear transform, which is actually a shear. Just as is shown in Fig. 1(c), the infinity-norm rotation along each side of the square is a simple shear transform.

\section{Piecewise Linear Implementation and Error Analysis}

All the infinity-norm rotations can be formulated with piecewise linear transforms in Euclidean space.

For the 2-D infinity-norm rotation, as shown in Fig. 2, we divide the $X Y$ plane into eight regions by 4 crossed dashed lines. In each region, the infinity-norm rotation is a linear transform, which is a shear transform or a product of a reflection and a shear transform. A 2-D infinity-norm rotation, analogously, is a shear and possibly an additional reflection with respect to the axes of symmetry $|x \pm y|=\sqrt{2}\|(x, y)\|_{\infty}$ to force the points sheared out of the boundary box to go back to the box, which can be realized by additions and assignments with if-else structures in programs. Using $R L$ to stand for runlength, the corresponding formula is as shown in (9) at the bottom of the page, where $a=\|(x, y)\|_{\infty}=\max \{|x|,|y|\}$.

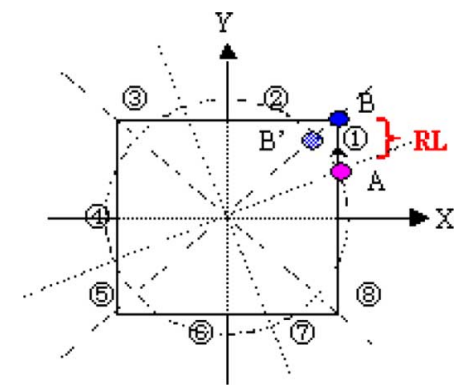

Fig. 2. Piecewise linearity of infinity-norm rotation.

Since an $n$-D 2-norm rotation can be factorized into a product of no more than $n(n-1) / 2$ 2-D 2-norm rotations [25], analogously, the $n$-D infinity-norm rotation can be implemented with no more than $n(n-1) / 22$-D infinity-norm rotations to approximate the correspondingly factored 2-norm rotations in 2-D.

If the input data are integers and the output are also required to be integers but the computation is not of integers, we should employ rounding arithmetic to obtain an integer runlength and just shear the points on the integer lattice of the data, and the inverse can be done by shearing back to the original position, thus the perfect reversibility is guaranteed.

For a 2-D vector $\mathbf{x}$, the runlength for integer-reversible infinity-norm rotation can be found as

$$
\text { runlength }=\left[\|\mathbf{x}\|_{\infty} \cdot \theta_{\infty}\right]
$$

where $[*]$ is a rounding operator, which can be round, ceil or floor. The infinity-norm rotation on the integer lattice can be formulated as (9) with runlength defined by (10).

Introducing rounding arithmetic into the transformation would result in rounding error and the error may also propagate with further transform computations. For the error resulted from quantization, we can estimate the bounds as follows.

If vector $\mathbf{x}$ is quantized as $\mathbf{x}^{\prime}=\mathbf{x}+\boldsymbol{\Delta}\left(\mathbf{x} \cdot \mathbf{x}^{\prime} \geq 0\right)$, then we have $\left\|R_{\infty}\left(\mathbf{x}^{\prime}, \theta_{\infty}\right)-R_{\infty}\left(\mathbf{x}, \theta_{\infty}\right)\right\|_{\infty} \leq\|\mathbf{\Delta}\|_{\infty}$ and $\left\|R_{\infty}\left(\mathbf{x}^{\prime}, \theta_{\infty}\right)-R_{\infty}\left(\mathbf{x}, \theta_{\infty}\right)\right\|_{2} \leq \sqrt{n}\|\mathbf{\Delta}\|_{\infty}$. To reduce the rounding error and its propagation, we generally expect the number of the factored transform matrices to be fewer and the infinity-norm of these matrices to be smaller.

In the following, we also use $R_{\infty}^{n}$ to denote the $n$-D infinitynorm rotation transform.

\section{INFINITY-NORM ROTATATION TRANSFORMS}

By analogy with the discrete cosine transforms (DCT), we propose Infinity-norm Rotation Transforms. DCT is widely ap-

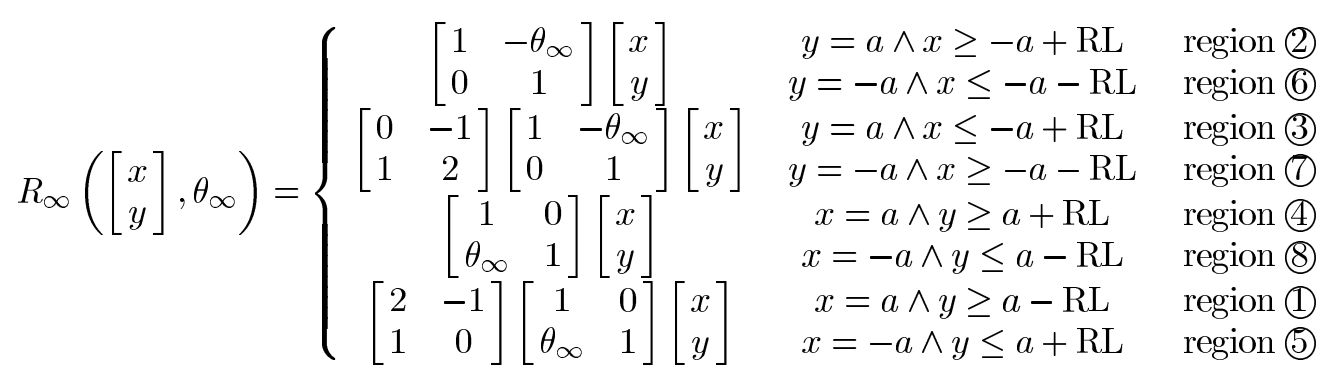




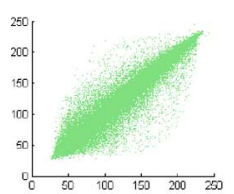

(a)

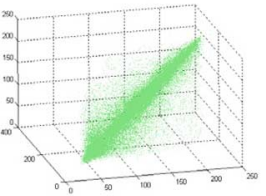

(d)

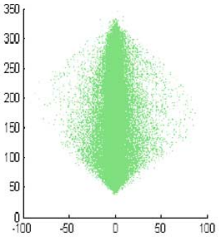

(b)

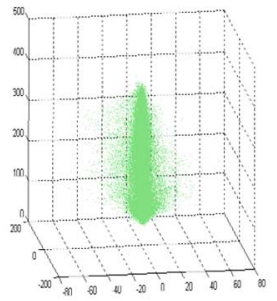

(e)

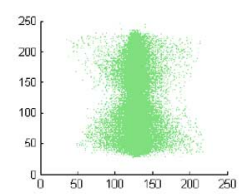

(c)

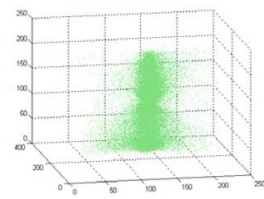

(f)
Fig. 3. Co-histograms of Lena: (a) original 2-D co-histogram, (b) after 2-point DCT, (c) after $R_{\infty}^{2}$, (d) original 3-D co-histogram, (e) after 3-point DCT, and (f) after $R_{\infty}^{2}$

plied in signal processing and performs very well, as DCT is the optimal transform for the first order Markov sequences when the correlation coefficients approximate 1 . Analogous to DCT, the proposed infinity-norm rotation transforms rotate signals in infinity-norm space, thus we can have similarly good performance in reducing the redundancy of signal representation.

The performance of transforms can be observed by comparing the $n$-D co-histograms of the original signal and the transformed signal. An $n$-D co-histogram is defined as follows.

Definition 4. n-D Co-Histogram of an Image: Taking every $n$ nonoverlapping neighboring pixels as a sample, $n$-D co-histogram of an image records the joint distribution of the samples, and shows the frequency of co-occurrences of every $n$ neighboring pixels in the image. Mathematically, an $n$-D co-histogram $H$ can be defined over an image $I$ as

$H\left(x_{1}, x_{2}, \cdots, x_{n}\right)=\sum_{p} \sum_{q}\left\{\prod_{(s, t) \in Q(p, q)} \delta\left(I(s, t)-x_{r}\right)\right\}$

where $\delta(*)$ is the Delta function, $I(*, *)$ is a pixel in the image, $x_{1}, x_{2}, \ldots, x_{n} \in[0,255], Q(p, q)$ is the $n$-pixel neighborhood of $(p, q)$, and $(s, t)$ is the coordinates of the $r$ th pixel in $Q(p, q)$.

The co-histogram reflects the relationship of neighboring pixels and order- $n$ statistical characteristics of changes in image pixel values. The $n$ neighboring pixels can be from 1-D sequences or 2-D blocks in an image. The $n$-D co-histogram can be used to analyze the inter-image or intra-image pixel relationships [17].

Take $512 \times 512$ Lena for an example. In Fig. 3(a) and (d), the samples are taken from every nonoverlapping couple and triple pixels in each row of Lena. The density of the samples betokens their frequencies. Due to the similarity of neighboring pixels in natural images, these samples primarily distribute along the diagonals of the co-histograms.
To investigate the performance of DCT, we apply the 2-point and 3-point DCT to couple and triple neighboring pixel values in each row of Lena. The 2-D and 3-D co-histograms of the DCT coefficients are given in Fig. 3(b) and (e), respectively. From mathematical representation of DCT and Fig. 3(b) and (e), we can see that the 2-point DCT matrix is equivalent to a counterclockwise 2-norm rotation by 45 degrees applied to the 2-D co-histogram, and that the 3-point DCT matrix is a rotation in 3-D space to turn the diagonal 3-D co-histogram parallel to an axis. The coefficients of DCT are decorrelated, because the co-histogram mainly parallel to at least one axis, which corresponds to the diagonal covariance matrices of the independent signals.

As a dynamic-range-preserving approximation of DCT, the infinity-norm rotation can act in a similar way. The 2-D and 3-D infinity-norm rotation transforms also turn the co-histograms mainly parallel to one axis though the distribution gets a bit wider in other directions. See Fig. 3(c) and (f). The definition of the infinity-norm rotation transforms is given as follows.

Definition 5. Infinity-Norm Rotation Transforms: Infinity-norm rotation transforms are the reversible and dynamic-range-preserving infinity-norm rotations, whose aim is to turn the co-histograms of the coefficients parallel to at least one axis.

Compared with DCT [Fig. 3(b) and (e)], after the 2-D and 3-D infinity-norm rotation transforms are applied to each row of Lena, the 2-D and 3-D co-histograms of the coefficients become parallel to axes as shown in Fig. 3(c) and (f). Fig. 3(c) and (f) also illustrate the multi-band decomposition ability of infinity-norm rotation transforms, which is very similar to that of wavelet transforms. After the rotation, the longest central axial line of the co-histogram gives the values of the equivalent low-frequency components, and other axial lines show the equivalent information of the high-frequency components. Thus, the neighboring pixels are well decorrelated after the infinity-norm rotation.

\section{IMPLEMENTATION AND EXAMPLES}

To implement an infinity-norm rotation transform, we can find a proper rotation angle by analogy with corresponding orthogonal transform, e.g., DCT.

Analogous to the 2-point DCT being a $45^{\circ}$ 2-norm rotation, we can find a rotation angle $\theta_{\infty}=1$ with (5) for $R_{\infty}^{2}$ and then use (9) for transform computation.

The 3-D and higher dimensional infinity-norm rotation transform can be implemented by using the 2-D rotation factorization of the corresponding DCT matrix [20]. For an infinity-norm rotation transform, the performance is very similar if implemented with different factorizations. The lower the dimension is, the fewer the factor matrices and the less the difference is, which will be shown in the experiments in next section.

Take $R_{\infty}^{3}$ as an example. The 3-point DCT has the two following factorizations, the coordinate plane factorization and cube-diagonal factorization.

1) The coordinate plane factorization in the first row of (12) is a product of a rotation around $Y$-axis and a rotation around $X$-axis. Then the corresponding infinity-norm 
rotation transform is a product of two infinity-norm rotations applied to $x, z$ coordinates and $y, z$ coordinates, respectively.

$$
\begin{aligned}
& {\left[\begin{array}{ccc}
\frac{\sqrt{3}}{3} & \frac{\sqrt{3}}{3} & \frac{\sqrt{3}}{3} \\
\frac{\sqrt{2}}{2} & 0 & -\frac{\sqrt{2}}{2} \\
\frac{\sqrt{6}}{6} & -\frac{\sqrt{6}}{3} & \frac{\sqrt{6}}{6}
\end{array}\right]} \\
& =\left[\begin{array}{ccc}
0 & \frac{\sqrt{3}}{3} & \frac{\sqrt{6}}{3} \\
1 & 0 & 0 \\
0 & -\frac{\sqrt{6}}{3} & \frac{\sqrt{3}}{3}
\end{array}\right] \cdot\left[\begin{array}{ccc}
\frac{\sqrt{2}}{2} & 0 & -\frac{\sqrt{2}}{2} \\
0 & 1 & 0 \\
\frac{\sqrt{2}}{2} & 0 & \frac{\sqrt{2}}{2}
\end{array}\right] \\
& =\left[\begin{array}{ccc}
\frac{\sqrt{2}}{2} & 0 & -\frac{\sqrt{2}}{2} \\
0 & 1 & 0 \\
\frac{\sqrt{2}}{2} & 0 & \frac{\sqrt{2}}{2}
\end{array}\right] \\
& \cdot\left(\left[\begin{array}{ccc}
\frac{\sqrt{2}}{2} & 0 & -\frac{\sqrt{2}}{2} \\
0 & 1 & 0 \\
\frac{\sqrt{2}}{2} & 0 & \frac{\sqrt{2}}{2}
\end{array}\right]^{-1} \cdot\left[\begin{array}{ccc}
0 & \frac{\sqrt{3}}{3} & \frac{\sqrt{6}}{3} \\
1 & 0 & 0 \\
0 & -\frac{\sqrt{6}}{3} & \frac{\sqrt{3}}{3}
\end{array}\right]\right. \\
& \left.\cdot\left[\begin{array}{ccc}
\frac{\sqrt{2}}{2} & 0 & -\frac{\sqrt{2}}{2} \\
0 & 1 & 0 \\
\frac{\sqrt{2}}{2} & 0 & \frac{\sqrt{2}}{2}
\end{array}\right]\right) \text {. }
\end{aligned}
$$

2) The cubic-diagonal factorization in the second row is a rotation around $Y$ axis and a cube-diagonal rotation (the three matrices in the round brackets). The corresponding implementation for $R_{\infty}^{3}$ is shown in Fig. 4 with two steps and explained below.

a) Cube-diagonal rotation.

Fig. 4(a) shows the cubic co-histogram of Lena which mainly distributes along the cube diagonal. A horizontal section slice of the co-histogram is shown in Fig. 4(b). The distribution on this slice is off-center. To imitate the function of the rightmost three factor matrices in the second row of (12), which rotates the diagonal to be parallel to $Z$ axis, we first move the 3-D samples along the highlighted boundaries of all the parallel rectangular slices [see Fig. 4(a)]. Then, we have a co-histogram mainly distributing parallel to $Z$-axis, and the centered distributions on the horizontal slices [see Fig. 4(c) and (d)]. For such a co-histogram, the $X Z$ coordinate correlation and $Y Z$ coordinate correlation are largely diminished. Although the diagonal slices are not square in $2-\mathrm{D}$, they are in the same isometry set in 3-D. Thus, the cube-diagonal rotation is also an infinity-norm rotation. The runlength on each slice could be determined by (10).

b) Plane-diagonal rotation.

After cube-diagonal rotation, the $X Y$ coordinates of the samples are still correlated, which is illustrated by the co-histogram diagonal in the horizontal slice [see Fig. 4(c) and (d)]. Thus, the infinity-norm rotations along the slice squares are needed to turn the distribution in horizontal slices to be axis aligned. See the highlighted boundaries of the horizontal slices in Fig. 4(c).

After the two above rotations, we have a co-histogram with two central axial lines parallel to the axes $Y$ and $Z$ of the co-

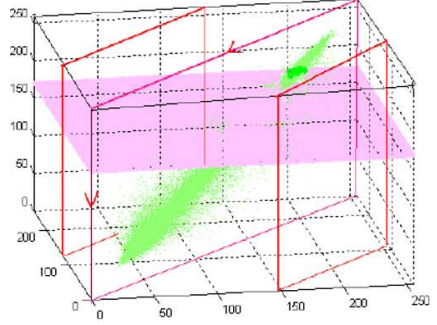

(a)

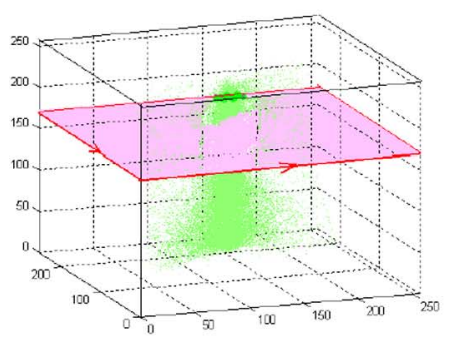

(c)

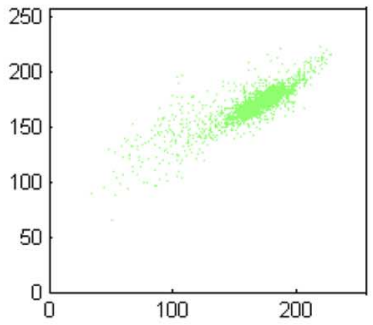

(b)

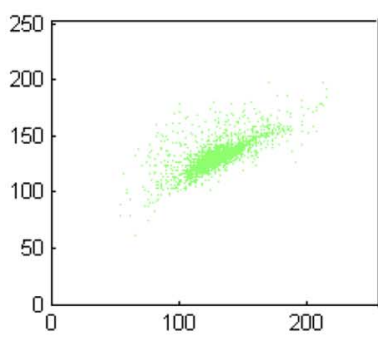

(d)

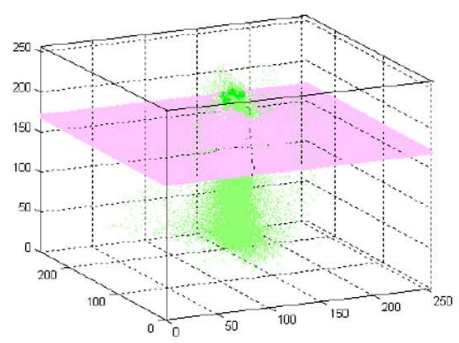

(e)

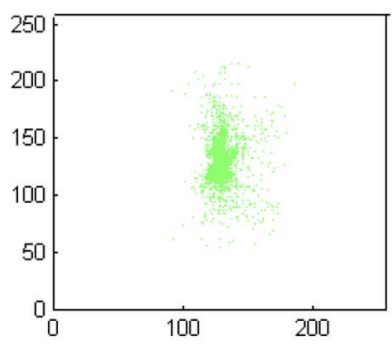

(f)
Fig. 4. 3-D infinity-norm rotation applied to Lena: (a) first cube-diagonal rotation, (b) a horizontal slice of (a), (c) further plane-diagonal rotation, (d) the slice of (c) (distribution centered), (e) co-histogram after $R_{\infty}^{3}$, and (f) the slice of, (e) (distribution centered and axes aligned.

ordinate system. Thus, the inter-pixel redundancy of the image pixels is greatly reduced [see Fig. 4(e) and (f)].

Implementations with different factorizations result in nearly the same entropy of coefficients in our experiments.

For general mathematical representation, for vector $(x, y, z)^{T}$, if $|z|=\|(x, y, z)\|_{\infty}=\max (|x|,|y|,|z|)$, the infinity-norm rotation transform can be formulated as a piecewise linear transform

$$
\left[\begin{array}{l}
x^{\prime} \\
y^{\prime} \\
z^{\prime}
\end{array}\right]=\left[\begin{array}{l}
x \\
y \\
z
\end{array}\right]-\operatorname{sign}(z)\left[\begin{array}{l}
1 \\
1 \\
0
\end{array}\right](|z|-|| x|-| y||)
$$

where $|*|$ is absolute operator, $|z|-|| x|-| y||$ gives the runlength, and $\left(x^{\prime}, y^{\prime}, z^{\prime}\right)^{T}$ is the transformed vector.

When the vector is sheared out of the dynamic range after moving a distance $D$, i.e., when $\|(x-\operatorname{sign}(z) D, y-$ $\operatorname{sign}(z) D) \|_{\infty}=|z|$ and $D<$ runlength, the vector should turn to shear along the adjacent vertical plane with the remaining runlength $|z|-|| x|-| y||-D$ :

$$
\left[\begin{array}{l}
x^{\prime} \\
y^{\prime} \\
z^{\prime}
\end{array}\right]=\left[\begin{array}{l}
x \\
y \\
z
\end{array}\right]-\operatorname{sign}(z)\left[\begin{array}{l}
0 \\
0 \\
1
\end{array}\right](|z|-|| x|-| y||-D) .
$$




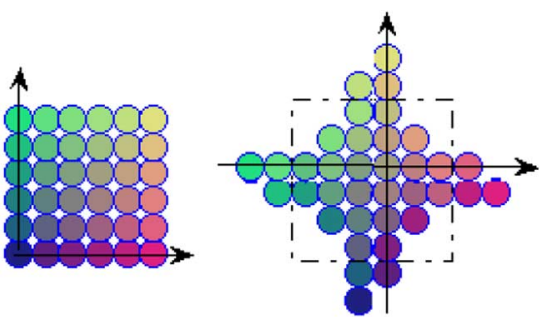

(a)

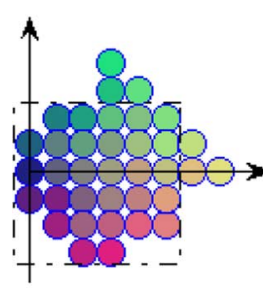

(d)

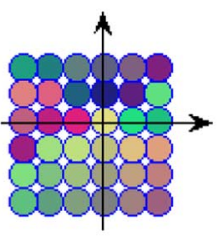

(g) (b)

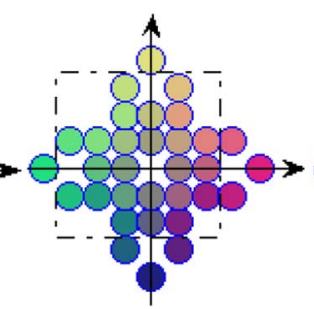

(e)

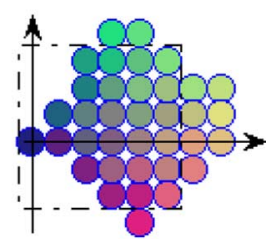

(h)

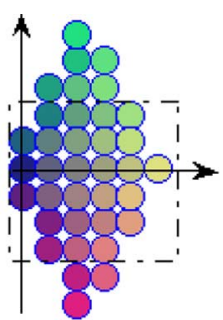

(c)

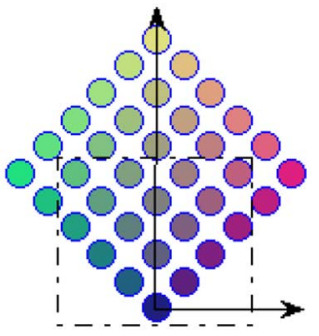

(f)

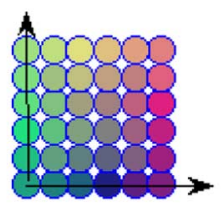

(i)
Fig. 5. Performance comparison with different transforms: (a) Original, (b) 1-norm rotation, (c) S transform, (d) RDCT, (e) DCT with rounding, (f) IntDCT, (g) CFM, (h) Modulo transform, and (i) Infinity-norm rotation.

If $|x|=\|(x, y, z)\|_{\infty}$ or $|y|=\|(x, y, z)\|_{\infty}$, the similar formula can be derived. It is obvious that the infinity-norm rotation transform is adaptive to the coordinates of the vectors to be rotated.

For higher-dimensional infinity-norm rotations, we can also use diagonal shears to implement by analogy with the diagonal factorizations of the corresponding orthogonal transform matrices.

\section{PERFormance COMPARISON With Other TRANSFORMS}

In this section, we first apply the proposed infinity-norm rotation transforms and a few other transforms to a typical 2-D pattern to show the differences between them, then we use histograms to compare their performance, and finally we list their computational complexity.

\section{A. Performance With a 2-D Pattern}

In order to clearly illustrate the performance differences between the infinity-norm rotation transforms and other transforms, we visualize the 2-point transforms applied to a $6 \times 6$ matrix pattern with integer coordinates [see Fig. 5(a)]. We take the coordinates of each sample in the pattern as a vector, and then transform the vector to a new one, as the new positions of the samples, which are illustrated in Fig. 5(b)-(i).

The tested transforms are (b) 1-norm rotation, (c) S transform [16], (d) integer reversible DCT by matrix factorization
(RDCT) [3], (e) 2-norm rotation (DCT) with direct rounding, (f) integer reversible DCT by scaling (IntDCT) [21], (g) CFH wavelet transform [12], and (h) modulo transform [19] and the proposed 2-D infinity-norm rotation transform.

The integer reversible transforms must keep the same number of samples, i.e., all the samples must appear in the pattern before and after transformation. There are overlapping and missing samples for the 2-norm rotation with rounding [see Fig. 5(e)], so the transform is not integer reversible. Sample overlapping or missing does not occur for all other tested transforms, so the rest transforms are all integer reversible. The dynamic range after the 1-norm rotation [see Fig. 5(b)], implemented by applying shearing along the rhomboids, has been expanded in both $X$ and $Y$ directions although it generates no "holes" and loses no samples. IntDCT [21] performs an counterclockwise rotation to the samples, and expands the dynamic range with a lot of "holes" [see Fig. 5(f)]. Its reversibility is guaranteed by expanding the dynamic range to construct a one-to-one mapping between the samples on integer coordinates. $\mathrm{S}$ transform, the integer implementation of Haar wavelet, takes a clockwise rotation, and eliminates the dynamic range expansion in the low-pass coefficients by omitting the least significant bits (LSB), which are the same as those of the high-pass coefficients. But the dynamic range of high-pass coefficients is nearly twice of the original. This LSB redundancy between the high and low-pass coefficients is also utilized in modulo transforms with butterfly structure. The performance of the DCT approximation $\mathrm{R}(4,3)$ for modulo transform is illustrated in Fig. 5(h). The transform matrix has a unit determinant, albeit with expanded dynamic range, which is similar to other orthonormal transforms. CFH is reversible, but the complementary coding and modular arithmetic violate the original neighboring relationship between the samples. Thus, when CFH is employed for lossy compression, the reconstructed samples from quantized coefficients may differ greatly from the original. The infinity-norm rotation transform is obviously piecewise linear, dynamic-range-keeping and one-to-one mapping [see Fig. 5(i)], because of the unchanged neighboring relationship of the samples.

For higher-dimensional transforms, it is difficult to visualize the transformation, but we can still use the statistical histograms to show the differences as in the next subsection.

\section{B. Histogram Comparison}

The main purpose to apply transforms for image coding is to decorrelate the neighboring pixels of images, or to compact the coefficient distribution so that the entropy can be reduced. In this subsection, we will present the histograms of coefficients for comparison after applying a few integer transforms, such as 4-point IntDCT with the least expansion factor of 2, 2-level integer 5/3 wavelet transform, 2-level CFH transform, 2-level modular $5 / 3$ transform (the modular $5 / 3$ wavelet is the modified dynamic-range-preserved version of $5 / 3$ wavelets with modular arithmetic, which is defined in the equation shown at the bottom of the next page), 4-point modulo transform, and our proposed 


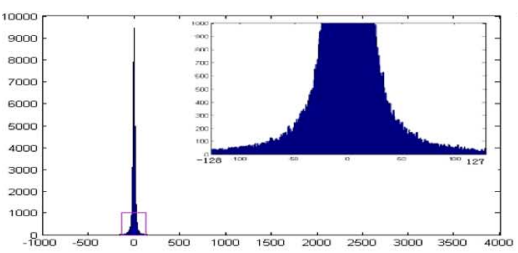

(a)

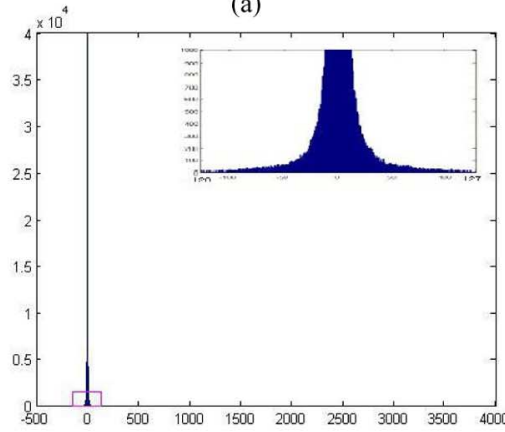

(c)

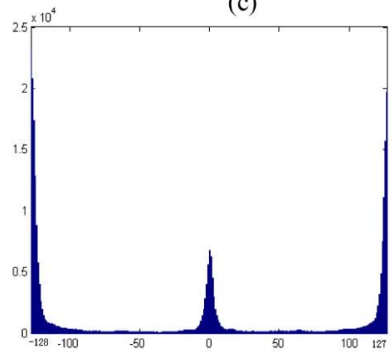

(e)

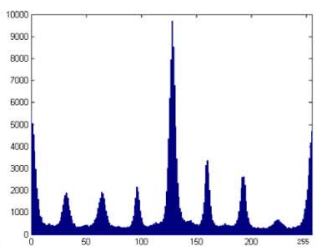

(b)

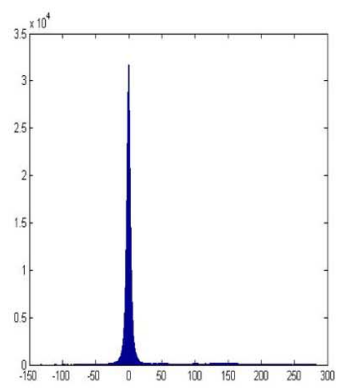

(d)

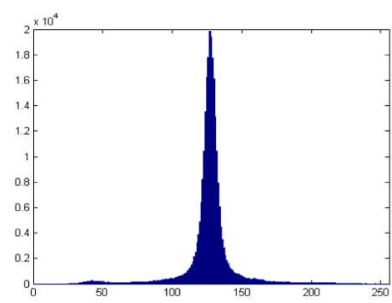

(f)
Fig. 6. Histograms of Lena after integer transforms: (a) IntDCT transform, (b) Modular 5/3 transform, (c) Modulo transform, (d) Integer 5/3 wavelet transform, (e) $\mathrm{CFH}$ transform, and (f) infinity-norm rotation $R_{\infty}^{4}$.

4-D infinity-norm rotation. The 4-point modulo transform to approximate the 4-point DCT is given by the following approximate matrix:

$$
T=\left[\begin{array}{cccc}
1 & 1 & 1 & 1 \\
\frac{12}{26} & \frac{5}{26} & -\frac{5}{26} & -\frac{12}{26} \\
\frac{1}{2} & -\frac{1}{2} & -\frac{1}{2} & \frac{1}{2} \\
-\frac{5}{26} & \frac{12}{26} & -\frac{5}{26} & \frac{5}{26}
\end{array}\right]
$$

The histograms are illustrated in Fig. 6. The sub-histogram of IntDCT is wider than that of the modulo transform in the same scale [see Fig. 6(a) and (c)], and the peak value of the histogram of IntDCT is less than that of the modulo transform, thus the compacting ability of IntDCT is not so satisfying as the modulo transform. It is also inferior to the infinity-norm rotation transform. The dynamic range of the modulo transform is so wide, since $\|T\|_{\infty}=4$, and its range is expanded by 16 times after $T$ is applied to rows and columns of the image. The histogram of integer 5/3 wavelet is centered well, and its dynamic range is expanded modestly. However, when with bitwidth constraint, the reconstructed images with integer $5 / 3$ have serious blocking
TABLE I

COMPUTATIONAL COMPLEXITY COMPARISON

\begin{tabular}{|c|c|c|c|}
\hline \hline Integer transforms & Addition & $\begin{array}{c}\text { Multiplication } \\
\text { or shifting }\end{array}$ & comparison \\
\hline INTDCT & $6 N^{2}$ & $16 N^{2}$ & --- \\
\hline 5/3 WAVELET & $10 N^{2}$ & $5 N^{2}$ & --- \\
\hline MODULAR 5/3 WAVELET & $10 N^{2}$ & $5 N^{2}$ & -- \\
\hline CFH WAVELET & $10 N^{2}$ & $5 N^{2}$ & -- \\
\hline MODULO TRANSFORM & $5 N^{2}$ & $2 N^{2}$ & --- \\
\hline$R_{\infty}^{4}$ & $3 N^{2} / 2$ & --- & $3 N^{2} / 2$ \\
\hline \hline
\end{tabular}

artifacts. The modular 5/3 transform presents multiple peaks, whereas the $\mathrm{CFH}$ gives two peaks after a circular shift by the complementary coding. The infinity-norm rotation transform, with exact 8 bits in $[0,255]$ for each coefficient, gives a compact and single-peak coefficient distribution, shown in Fig. 6(f). More importantly, the histogram similarity between integer 5/3 wavelet transform and the infinity-norm rotation means that the wavelet-based JPEG 2000 framework, such as progressive rate allocation, can be utilized directly to accommodate the infinitynorm rotation, rewarding similar or just little inferior compression performance, which is shown in the next section.

\section{Computational Complexity}

The computational complexity of integer transforms for $N \times$ $N$ images is compared in Table I. With the infinity-norm rotation transform $R_{\infty}^{4}$ is with the least computational cost, only comparison and additions are needed. For $512 \times 5128$-bit gray images, with Matlab 7.0, the elapsed time for infinity-norm rotation transform is $9.80 \mathrm{~s}$, whereas that for CFH is $19.00 \mathrm{~s}$.

In summary, the proposed infinity-norm rotation transform is integer reversible and fast, preserves the dynamic range, gives a compact output, and performs well for data decorrelation.

\section{APPliCATION IN IMAge CODING AND EXPERIMENTAL RESULTS}

In this section, we apply the infinity-norm rotation transforms to image coding. Compared with other tested integer transforms, the infinity-norm rotation transform is effective in both lossy and lossless image coding under the fixed bitwidth constraint. These properties cannot be simultaneously realized with other tested transforms. Note that for images with data range in [0, $255]$, the origin should be firstly shifted to 127.5 , the center of the dynamic range of the pixels, and then shifted back.

We test image coding in our experiments with 2-D, 3-D, 4-D, and 8-D infinity-norm rotation transforms and other seven types of reversible transforms (see Table II):

1) $S$ transform (denoted as $S$ );

2) 2-point Integer DCT by matrix factorization (denoted as RDCT2);

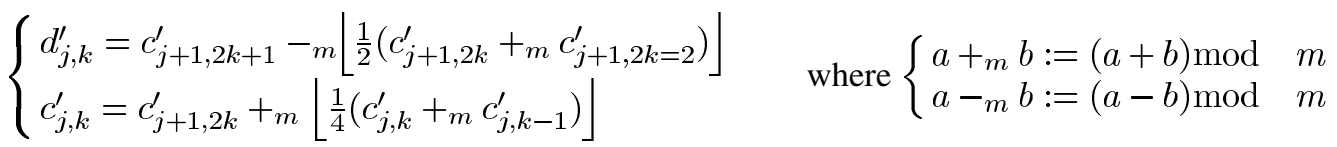


TABLE II

ENTROPY COMPARISON BETWEEN INTEGER TRANSFORMS FOR LOSSLESS COMPRESSION

\begin{tabular}{|c|c|c|c|c|c|c|c|c|c|c|c|}
\hline \hline Image & $\mathrm{S}$ & RDCT2 & INTDCT4 & $5 / 3$ & CFH & MW & MT & $R_{\infty}^{2}$ & $R_{\infty}^{3}$ & $R_{\infty}^{4}$ & $R_{\infty}^{8}$ \\
\hline Barbara & 6.70 & 6.79 & 7.66 & 5.49 & 6.30 & 7.45 & 5.82 & 6.45 & 6.10 & 6.00 & 6.03 \\
\hline Lena & 6.18 & 6.22 & 7.12 & 4.85 & 5.80 & 7.26 & 5.31 & 5.97 & 5.52 & 5.38 & 5.42 \\
\hline Boat & 6.19 & 6.25 & 7.24 & 5.01 & 5.82 & 7.25 & 5.42 & 6.10 & 5.66 & 5.50 & 5.54 \\
\hline Jet & 5.90 & 5.75 & 7.04 & 4.75 & 6.13 & 6.99 & 5.23 & 5.55 & 5.30 & 5.23 & 5.33 \\
\hline Mandrill & 7.36 & 7.09 & 8.73 & 6.50 & 6.84 & 7.87 & 6.91 & 6.89 & 6.74 & 6.73 & 6.80 \\
\hline Goldhill & 6.49 & 6.54 & 7.59 & 5.31 & 6.25 & 7.54 & 5.80 & 6.26 & 5.88 & 5.74 & 5.79 \\
\hline Average & 6.47 & 6.44 & 7.56 & 5.32 & 6.19 & 7.39 & 5.73 & 6.20 & 5.87 & 5.76 & 5.82 \\
\hline \hline
\end{tabular}

3) 4-point IntDCT [21] (denoted as IntDCT4);

4) two-level decomposition with integer $5 / 3$ wavelet (denoted as $5 / 3$;

5) two-level decomposition with CFH [12] 5/3 wavelet;

6) modular $5 / 3$ wavelet (denoted as MW);

7) modulo transform [19] which approximates 4-point DCT (denoted as MT);

8) rotate every pair of pixels with $R_{\infty}^{2}$ in each row of the image, and then in each column (denoted as $R_{\infty}^{2}$ );

9) rotate every triple pixels with two successive $R_{\infty}^{2}$ with the coordinate plane factorization in each row of the image, and then in each column (denoted as $R_{\infty}^{3}$ );

10) rotate every four pixels with three successive $R_{\infty}^{2}$ in each row of the image, and then in each column to implement the 4-D infinity-norm rotation transform (denoted as $R_{\infty}^{4}$ );

11) rotate every eight pixels with seven successive $R_{\infty}^{2}$ in each row of the image, and then in each column to implement the 8-D infinity-norm rotation transform (denoted as $R_{\infty}^{8}$ ).

These transforms are applied to both the rows and columns of the images. After transformation, the coefficient distributions are compact, and their entropies are calculated with all the transformed data and listed in Table II for lossless coding comparison. We have the following findings from Table II.

1) The ability of 2-D infinity-norm rotation to eliminate redundancy is superior to $\mathrm{S}$ transform and RDCT, since the dynamic range constraint results in fewer number of symbols, which rewards with lower entropy.

2) The reversible transforms, IntDCT $4,5 / 3$ and MT, result in expanded dynamic ranges. If under constant bitwidth constraint, they are not reversible anymore, and reconstructed images have serious blocking artifacts.

3) Although rotation of IntDCT4 reduces the inter-pixel redundancy partially, representation redundancy brought by expanded dynamic range offsets this merit. Therefore, its entropy performance in Table II is not satisfying, and much less desirable than other integer transforms for compression.

4) Without considering the dynamic range expansion, 5/3 wavelet transform performs the best with the lowest entropy. The next winners are $R_{\infty}^{4}$ and MT. The infinity-norm rotation gives satisfying compression performance, more importantly, with constant dynamic ranges.

For lossy image coding, we apply the uniform quantization scheme to the coefficients after the infinity-norm rotation transform and $\mathrm{CFH}$ transform:

$$
I(i, j)=\text { floor }\left(I(i, j) / 2^{n}\right) \cdot 2^{n} .
$$

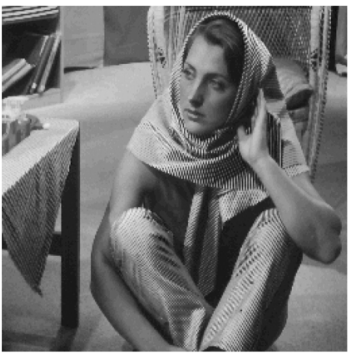

(a)

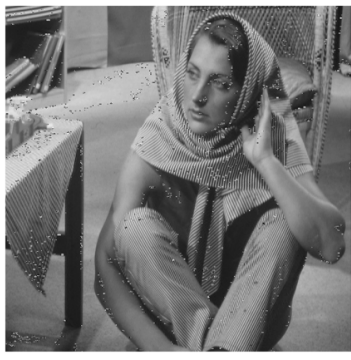

(b)
Fig. 7. Reconstructed images after lossy compression: (a) lossy coding with $R_{\infty}$ and (b) lossy coding with CFH.

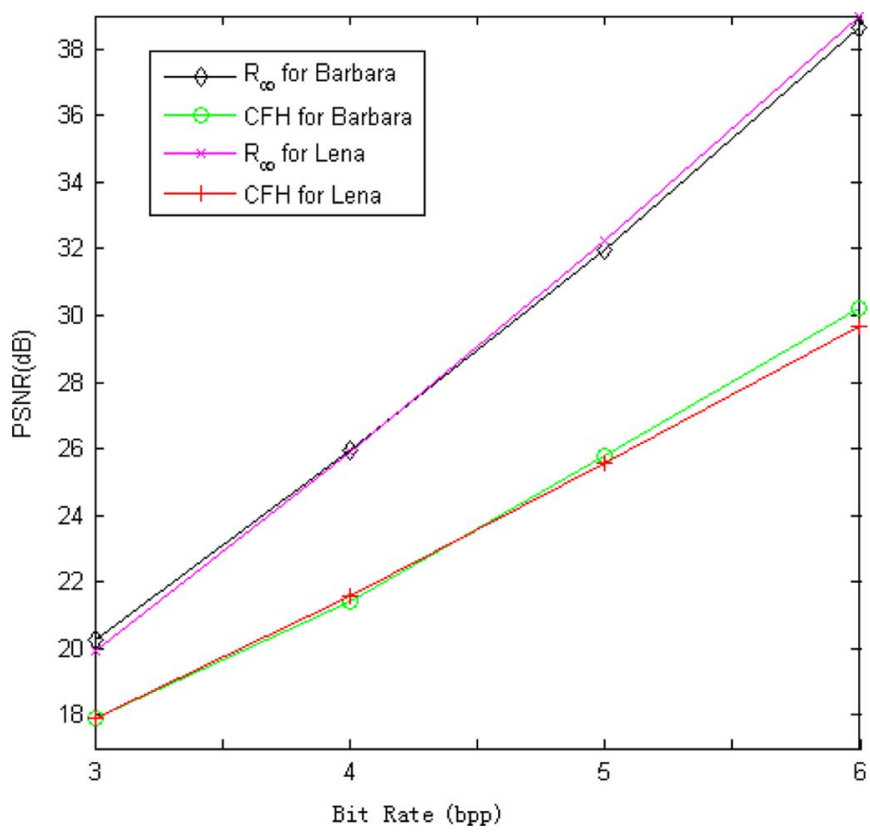

Fig. 8. Lossy coding performance comparison between $R_{\infty}^{4}$ and CFH.

The image results with Barbara are given in Fig. 7, and the PSNR results are shown in Fig. 8. The CFH is only applicable to lossless compression, and with the salt-and-pepper artifacts for lossy compression, whereas the piecewise linearity of the infinity-norm rotation transforms makes lossy coding feasible without salt-and-pepper noise. Therefore, the infinity-norm rotation transforms are suitable for unified lossy/lossless transform coding and lossy reproduction (progressive or nonprogressive) from lossless compressed data as wavelet transforms. 
TABLE III

AVERAGE BIT RATES FOR LOSSLESS COMPRESSION

\begin{tabular}{|c|c|c|c|c|}
\hline \hline Method & RCT & RDCT & SHIRCT & Infinity-norm \\
\hline $\begin{array}{c}\text { Average bit } \\
\text { rate (bpp) }\end{array}$ & 12.39 & 11.70 & 12.00 & 11.87 \\
\hline \hline
\end{tabular}

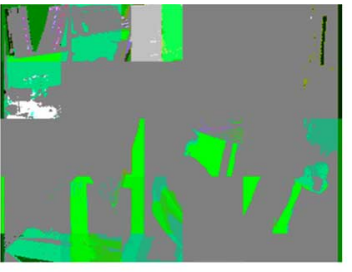

(a)

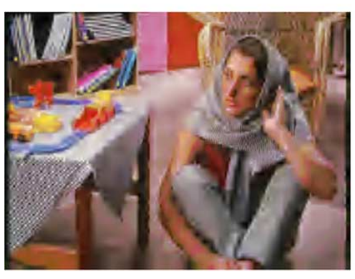

(b)

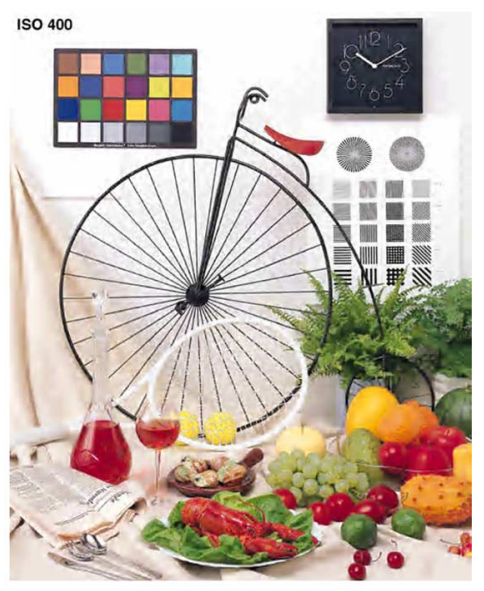

(c)
Fig. 9. Reconstructed images with 8 bitwidth at 0.0625 bpp: (a) fixed 8-bit color transform in JPEG2000, (b) $R_{\infty}^{3}$, and (c) $R_{\infty}^{3}$.

We also use $R_{\infty}^{3}$ as lossless color transform in JPEG2000 coding framework, and compare its performance with other color transforms, i.e., RCT [26], RDCT and SHIRCT [27] in Table III. We used 26 color images for our experiments, Bike, Woman, Airplane, Baboon, Barbara, Boats, Couple, Fruits, Frymire, Girl, Girl2 Girl3, Goldhill, House, Lena, Monarch, Peppers, Sail, Sailboat, Serreno, Splash, Target, Tiffany, Tree, Tulips, and Zelda. For $R_{\infty}^{3}$ applied to image coding as lossy color transform, the reconstructed images are shown in Fig. 9(b) and (c) without false color problem, whereas the reconstructed image Barbara has severe blocking artifacts using the fixed 8-bit truncated coefficients after color transform in the framework of JPEG2000, which is shown in Fig. 9(a).

To sum up, the infinity-norm rotation transform is reversible, piecewise linear and dynamic-range-preserving, thus it is suitable for both lossy and lossless coding.

\section{CONCLUSION}

In this paper, we propose a new family of integer-reversible, piecewise-linear and dynamic-range-preserving transformsinfinity-norm rotation transforms, which have applications in image coding and reversible data hiding [23], [24]. The constant dynamic range allows fast fixed-point and in-place calculation, and its computational complexity is small. It needs only limited buffer, fixed point arithmetic unit and fixed bitwidth channels. As a new class of simple piecewise linear integer transforms, the proposed infinity-rotation transforms may find more promising applications in image processing in the future.

Besides exploring the applications, our future work for the infinity-norm rotation transform will be concentrated on developing a more systematic theory for Multiscale Geometric Analysis (MGA).

\section{REFERENCES}

[1] W. Sweldens, "The lifting scheme: A new philosophy in biorthogonal wavelet constructions," in Proc. SPIE, San Diego, CA, Sep. 1995, vol. 2569, pp. 68-79.

[2] P. Hao, "Customizable triangular factorizations of matrices," Linear Algebra Appl., vol. 382, pp. 135-154, 2004.

[3] P. Hao and Q. Shi, "Matrix factorizations for reversible integer mapping," IEEE Trans. Signal Process., vol. 49, no. 10, pp. 2314-2324, Oct. 2001.

[4] H.-Y. Jung and R. Prost, "Lossless subband coding system based on rounding transform," IEEE Trans. Signal Process., vol. 46, no. 9, pp. 2535-2540, Sep. 1998.

[5] I. Daubechies and W. Sweldens, "Factoring wavelet transforms into lifting steps," J. Fourier Anal. Appl., vol. 4, pp. 247-269, 1998.

[6] W. Sweldens, "The lifting scheme: A construction of second generation wavelets," J. Math. Anal., vol. 29, pp. 511-546, 1997.

[7] Y. J. Chen, S. Oraintara, and T. Nguyen, "Video compression using integer DCT," in Proc. IEEE Int. Conf. Image Process., Vancouver, BC, Canada, 2000, vol. 2, pp. 844-847.

[8] J. Liang and T. D. Tran, "Fast multiplierless approximation of the DCT with the lifting scheme," in Proc. 23rd SPIE App. Dig. Image Process. , Aug. 2000, vol. 4115, pp. 384-395.

[9] S. Oraintara, Y.-J. Chen, and T. Nguyen, "Integer fast Fourier transform," IEEE Trans. Signal Process., vol. 50, no. 3, pp. 607-618, Mar. 2002.

[10] M. Adams and F. Kossentini, "On the relationship between overlapping rounding transform and lifting frameworks for reversible subband transforms," IEEE Trans. Signal Process., vol. 48, no. 1, pp. 261-266, Jan. 2000.

[11] A. Kiely and M. Klimesh, "The ICER progressive wavelet image compressor," Interplanetary Network Progr. Rep., vol. 42, no. 155, pp. 1-46, Nov. 2003.

[12] H. Chao, P. Fisher, and Z. Hua, "An approach to integer wavelet transformations for lossless image compression," in Advances in Computational Mathematics Lecture Notes in Pure and Applied Mathematics. New York: Marcel Dekker, 1999, vol. 202, pp. 13-38.

[13] J. G. Senecal, M. A. Duchaineau, and K. I. Joy, "Reversible $n$-bit to $n$-bit integer Haar-like transforms," in Proc. Data Compression Conf., Snowbird, UT, 2004, p. 564.

[14] J. G. Senecal, P. Lindstrom, M. A. Duchaineau, and K. I. Joy, "An improved $n$-bit to $n$-bit reversible Haar-like transform," in Proc. 12th Pacific Conf. Computer Graphics Applications, Seoul, Korea, Oct. 2004, pp. 371-380.

[15] S. D. Rane and G. Sapiro, "Evaluation of JPEG-LS, the new lossless and controlled-lossy still image compression standard, for compression of high-resolution elevation data," IEEE Trans. Geosci. Remote Sens., vol. 39 , no. 10, pp. 2298-2306, 2001.

[16] A. Said and W. A. Pearlman, "An image multiresolution representation for lossless and lossy compression," IEEE Trans. Image Process., vol. 5, no. 9, pp. 1303-1310, 1996.

[17] P. Hao, C. Zhang, and A. Dang, "Co-histogram and image degradation evaluation," in Proc. IEEE Int. Conf. Intelligent Automation Robotics (ICIAR), 2004, pp. 195-203

[18] P. Hao and Q. Shi, "Proposal of reversible integer implementation for multiple component transforms," ISO/IET JTC1/SC29/WG1 N 1720, Jun. 26, 2000.

[19] S. Srinivasan, "Modulo transforms-An alternative to lifting," IEEE Trans. Signal Process., vol. 54, no. 5, pp. 1864-1874, May 2006.

[20] G. Plonka and M. Tasche, "Invertible integer DCT algorithms," Appl. Comput. Harmon. Anal., vol. 15, pp. 70-88, 2003.

[21] G. Plonka, "A global method for invertible integer DCT and integer wavelet algorithms," Appl. Comput. Harmon. Anal., vol. 16, pp. 90-110, Mar. 2004.

[22] A. E. Taylor and D. C. Lay, Introduction to Functional Analysis, 2nd ed. New York: Wiley, p. c1980.

[23] L. Yang, P. Hao, and C. Zhang, "Progressive reversible data hiding by symmetrical histogram expansion with piecewise linear Haar transform," Proc. IEEE Int. Conf. Acoustics, Speech, Signal Processing (ICASSP), pp. 265-268, Apr. 20-22, 2007.

[24] L. Yang and P. Hao, "Infinity-norm rotation for reversible data hiding," Proc. IEEE Int. Conf. Image Processing (ICIP), pp. 245-248, Sep. 2007.

[25] N. J. Vilenkin, "Special functions and the theory of group representations," in Translations of Mathematical Monographs. Providence, RI: Amer. Math. Soc., 1983, vol. 22.

[26] D. S. Taubman and M. W. Marchellin, JPEG2000 Image Compression Fundamentals, Standards and Practice. Norwell, MA: Kluwer, 2002, 2nd printing. 
[27] P. Hao and Q. Shi, "Comparative study of color transforms for image coding and derivation of integer reversible color transform," in Proc. 15th Int. Conf. Pattern Recognition (ICPR), Sep. 3-8, 2000, vol. 3, pp. 224-227.

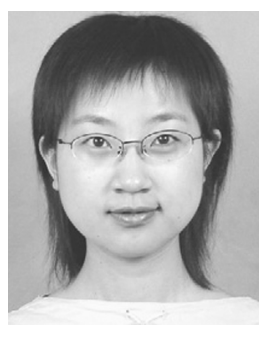

Lei Yang received the B.Sc. degree in computer science from the Beijing Information Science and Technology University, Beijing, China, and the M.E. degree in electrical engineering from Peking University, Beijing, China, in 2004 and 2008, respectively. She is currently working towards the Ph.D. degree in electrical and computer engineering at University of Florida, Gainesville, FL.

Her research interests include signal processing, image coding, multimedia watermarking, machine learning, and computer vision.

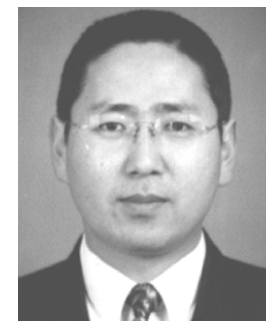

Pengwei Hao (M'98) received the B.Sc. degree in computer science and the M.Sc. degree in computer graphics from Northwestern Polytechnical University, Xi'an, China, in 1988 and 1994, respectively, and the Ph.D. degree in image processing from the Institute of Remote Sensing Applications, Chinese Academy of Science, Beijing, China, in 1997.

From 1997 to 1999, he was a Lecturer at the Center for Information Science, Peking University, Beijing, China. He was a Visiting Researcher for three months with the Center for Vision, Speech, and Signal Processing, University of Surrey, Surrey, U.K., in 2000, and a Visiting Researcher for three months with the School of Information Technology and Engineering (SITE), University of Ottawa, Canada in 2008. In 2002, he was appointed to Lecturer at Queen Mary, University of London, London, U.K. He is also currently an Associate Professor with the Center for Information Science, Peking University, China. His research interests include data and image compressing, data hiding, signal sampling and reconstruction, and integer transforms. 